\title{
Kaons and antikaons in strong magnetic fields
}

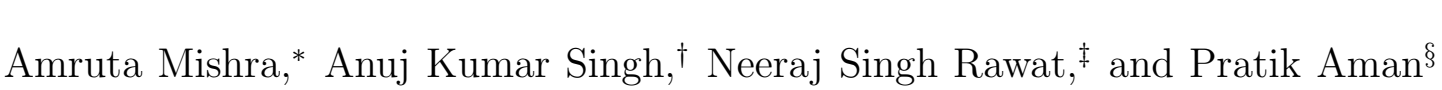 \\ Department of Physics, Indian Institute of Technology, \\ Delhi, Hauz Khas, New Delhi - 110 016, India
}

\begin{abstract}
The in-medium masses of the kaons and antikaons in strongly magnetized asymmetric nuclear matter are studied using a chiral SU(3) model. The medium modifications of the masses of these open strange pseudoscalar mesons arise due to their interactions with the nucleons and scalar mesons within the model. The effects of anomalous magnetic moments (AMM) of the nucleons are taken into consideration in the present study and these are seen to be large at high magnetic fields and high densities. The isospin effects are appreciable at high densities. The density effects are observed to be the dominant medium effects, as compared to the effects from magnetic field and isospin asymmetry.
\end{abstract}

\footnotetext{
*Electronic address: amruta@physics.iitd.ac.in

${ }^{\dagger}$ Electronic address: anuj0630@gmail.com

‡Electronic address: neerajrawat377@gmail.com

$\S$ Electronic address: pratikaman.atom@gmail.com
} 


\section{INTRODUCTION}

The topic of hadrons in hot and/or dense matter has been a subject of extensive research due to its relevance in relativistic heavy ion collision experiments in various high energy particle accelerators, as well as, for study of bulk properties of astrophysical objects, e.g., neutron stars. There are huge magnetic fields produced in noncentral ultrarelativistic heavy ion collision experiments [1-4]. There is also natural occurrence of large magnetic fields in magnetars. The study of the effects of magnetic fields, in addition to the effects of high density and/or temperature on the properties of hadrons has emerged as an intense research topic in strong interaction physics in the recent years. The initial system of the heavy ion collisions has isospin asymmetry (as $N \neq Z$ in the colliding nuclei) and hence it is important to study the effects of the isospin asymmetry on the properties of the hadrons. The various approaches used for the study of in-medium properties of hadrons are the QCD sum rule approach [5, 6], the effective hadronic models, e.g., Quantum Hadrodynamics (QHD) model [7, 8] and its generalizations, the Quark meson coupling (QMC) model [9, 10] where the quarks/antiquarks inside of the hadron interact via exchange of scalar and vector mesons, and effective hadronic models based on low energy properties of QCD, e.g., chiral symmetry breaking, as well as, coupled channel approach [11-16].

In the present work, using a chiral SU(3) model [17, 18], the medium modifications of the masses of the kaons and antikaons in asymmetric nuclear matter in the presence of strong magnetic fields are studied. The proton has contributions from the Landau energy levels in the presence of an external magnetic field. The medium modifications of kaons and antikaons arise due to the interaction with the nucleons and scalar mesons in the magnetized nuclear matter, within the chiral SU(3) model. The masses of the charged $K^{ \pm}$mesons have additional positive shift in the magnetized medium, arising from the direct interaction of these mesons with the magnetic field. The model has been used to study, at zero magnetic field, the kaons and antikaons in isospin asymmetric nuclear (hyperonic) matter [18 22], finite nuclei [17], vector mesons [23, 24], neutron stars [25]. The in-medium masses of open (strange) charm [26-30], open bottom mesons [31, 32], charmonium [29, 33] and bottomonium states [34] have also been studied within the hadronic model by generalizing the model to the charm and bottom sectors. The partial decay widths of the charmonium states to $D \bar{D}$ pair [29, 35 40], as well as, 
of bottomonium states to $B \bar{B}$ [41] in the hadronic medium have been studied, from the mass modifications of these hidden and open heavy flavour mesons. Recently, the effects of magnetic fields have been considered on the light vector mesons [42] as well as the heavy flavour mesons [43 47], through the coupling of the nucleons with the uniform magnetic field, within the chiral

effective model. The effects of the anomalous magnetic moments of the nucleons [48 55] on the mass modifications of these hadrons are also studied.

The outline of the paper is as follows : In section II, we describe briefly the chiral $S U(3)$ model used to investigate the mass modifications of the kaons and antikaons in asymmetric nuclear matter in the presence of strong magnetic fields. Section III discusses the results of the medium modifications of the masses of these open strange pseudoscalar mesons in the magnetized asymmetric nuclear matter. In section IV, we summarize the findings of the present investigation.

\section{THE CHIRAL SU(3) MODEL}

We use a chiral $S U(3)$ model [17] to obtain the in-medium masses of the kaons and antikaons in magnetized nuclear matter. The model is based on the nonlinear realization of chiral symmetry [56 59] and broken scale invariance [17, 18, 23]. The concept of broken scale invariance leading to the trace anomaly in QCD, $\theta_{\mu}^{\mu}=\frac{\beta_{Q C D}}{2 g} G^{a}{ }_{\mu \nu} G^{\mu \nu a}$, where $G_{\mu \nu}^{a}$ is the gluon field strength tensor of QCD, is simulated in the effective Lagrangian at tree level through the introduction of a logarithmic potential in terms of a scalar dilaton field, $\chi[60-62]$.

The Lagrangian density of the chiral SU(3) model, in the presence of magnetic field, is given as [17, 43,44 ]

$$
\mathcal{L}=\mathcal{L}_{\text {kin }}+\sum_{W=X, Y, V, \mathcal{A}, u} \mathcal{L}_{B W}+\mathcal{L}_{\text {vec }}+\mathcal{L}_{0}+\mathcal{L}_{\text {scalebreak }}+\mathcal{L}_{S B}+\mathcal{L}_{\text {mag }}^{B \gamma}
$$

where, $\mathcal{L}_{\text {kin }}$ corresponds to the kinetic energy terms of the baryons and the mesons, $\mathcal{L}_{B W}$ contains the baryon-meson interactions, $\mathcal{L}_{v e c}$ describes the dynamical mass generation of the vector mesons via couplings to the scalar fields and contains additionally quartic self-interactions of the vector fields, $\mathcal{L}_{0}$ contains the meson-meson interaction terms $\mathcal{L}_{\text {scalebreak }}$ is a scale invariance breaking logarithmic potential, $\mathcal{L}_{S B}$ describes the explicit chiral symmetry breaking and $\mathcal{L}_{\text {mag }}^{B \gamma}$ describes the effects due to the magnetic field through the coupling of the baryons to the 
electromagnetic field. The kinetic energy terms are given as

$$
\begin{aligned}
\mathcal{L}_{k i n} & =i \operatorname{Tr} \bar{B} \gamma_{\mu} D^{\mu} B+\frac{1}{2} \operatorname{Tr} D_{\mu} X D^{\mu} X+\operatorname{Tr}\left(u_{\mu} X u^{\mu} X+X u_{\mu} u^{\mu} X\right)+\frac{1}{2} \operatorname{Tr} D_{\mu} Y D^{\mu} Y \\
& +\frac{1}{2} D_{\mu} \chi D^{\mu} \chi-\frac{1}{4} \operatorname{Tr}\left(\tilde{V}_{\mu \nu} \tilde{V}^{\mu \nu}\right)-\frac{1}{4} \operatorname{Tr}\left(\mathcal{A}_{\mu \nu} \mathcal{A}^{\mu \nu}\right)-\frac{1}{4} \operatorname{Tr}\left(F_{\mu \nu} F^{\mu \nu}\right)
\end{aligned}
$$

where, $B$ is the baryon octet, $X$ is the scalar meson multiplet, $Y$ is the pseudoscalar chiral singlet, $\chi$ is the scalar dilaton field, $\tilde{V}_{\mu \nu}=\partial_{\mu} \tilde{V}_{\nu}-\partial_{\nu} \tilde{V}_{\mu}, \mathcal{A}_{\mu \nu}=\partial_{\mu} \mathcal{A}_{\nu}-\partial_{\nu} \mathcal{A}_{\mu}$, and $F_{\mu \nu}=$ $\partial_{\mu} A_{\nu}-\partial_{\nu} A_{\mu}$, are the field strength tensors of the renormalised vector meson multiplet, $\tilde{V}^{\mu}$, the axial vector meson multiplet $\mathcal{A}^{\mu}$ and the photon field, $A^{\mu}$. In Eq. (2), $u_{\mu}=-\frac{i}{4}\left[\left(u^{\dagger}\left(\partial_{\mu} u\right)-\right.\right.$ $\left.\left.\left(\partial_{\mu} u^{\dagger}\right) u\right)-\left(u\left(\partial_{\mu} u^{\dagger}\right)-\left(\partial_{\mu} u\right) u^{\dagger}\right)\right]$, where, $u=\exp \left[\frac{i}{\sigma_{0}} \pi^{a} \lambda^{a} \gamma_{5}\right]$. The covariant derivative of a field $\Phi(\equiv B, X, Y, \chi)$ reads $D_{\mu} \Phi=\partial_{\mu} \Phi+\left[\Gamma_{\mu}, \Phi\right]$, with $\Gamma_{\mu}=-\frac{i}{4}\left[\left(u^{\dagger}\left(\partial_{\mu} u\right)-\left(\partial_{\mu} u^{\dagger}\right) u\right)+\left(u\left(\partial_{\mu} u^{\dagger}\right)-\right.\right.$ $\left.\left.\left(\partial_{\mu} u\right) u^{\dagger}\right)\right]$. The interaction of the baryons with the meson, $W$ (scalar, pesudoscalar, vector, axialvector meson) given by $\mathcal{L}_{B W}$, as well as the terms $\mathcal{L}_{\text {vec }}, \mathcal{L}_{0}, \mathcal{L}_{\text {scalebreak }}, \mathcal{L}_{S B}$ have been described in detail in Ref. [17]. The term $\mathcal{L}_{\text {mag }}^{B \gamma}$, describing the interacion of the baryons with the electromagnetic field is given as [43, 44]

$$
\mathcal{L}_{\text {mag }}^{B \gamma}=-\bar{\psi}_{i} q_{i} \gamma_{\mu} A^{\mu} \psi_{i}-\frac{1}{4} \kappa_{i} \mu_{N} \bar{\psi}_{i} \sigma^{\mu \nu} F_{\mu \nu} \psi_{i}
$$

where, $\psi_{i}$ corresponds to the $i$-th baryon. The tensorial interaction of baryons with the electromagnetic field given by the second term in the above equation is related to the anomalous magnetic moments of the baryons [48 55]. We choose the magnetic field to be uniform and along the z-axis, and take the vector potential to be $A^{\mu}=(0,0, B x, 0)$.

The calculations in the present work of study of the kaons and antikaons in the magnetized nuclear matter are carried out in the mean field approximation, where the meson fields are replaced by their expectation values. In addition to using the mean field approximation, where the meson fields are replaced by their expectation values, we also use the approximations that $\bar{\psi}_{i} \psi_{j}=\delta_{i j}\left\langle\bar{\psi}_{i} \psi_{i}\right\rangle \equiv \delta_{i j} \rho_{i}^{s}$ and $\bar{\psi}_{i} \gamma^{\mu} \psi_{j}=\delta_{i j} \delta^{\mu 0}\left\langle\bar{\psi}_{i} \gamma^{0} \psi_{i}\right\rangle \equiv \delta_{i j} \delta^{\mu 0} \rho_{i}$, where, $\rho_{i}^{s}$ and $\rho_{i}$ are the scalar and number density of fermion of species, $i$ (neutron and proton in the present investigation). We use the frozen glueball approximation, i.e., we fix $\chi=\chi_{0}$, the vacuum value of the dilaton field. This is due to the reason that the medium modification of the dilaton field in nuclear medium is observed to be negligible as compared to the medium changes of the scalar fields, $\sigma, \zeta$ and $\delta$. The coupled equations of motions of these scalar fields are derived from the 
Lagrangian density of the chiral SU(3) model, and, are solved to obtain the values of these fields in the asymmetric nuclear medium in the presence of magnetic field. The number and scalar densities of the proton have contributions from the Landau energy levels and the neutrons have contributions to their number and scalar densities due to the anomalous magnetic moment, in the presence of a magnetic field [43, 44]. The expresssions for the number and scalar densities of the proton in the presence of a uniform magnetic field (chosen to be along z-direction) and accounting for the anomalous magnetic moments for the nucleons are given as [43, 44, 50, 51]

$$
\rho_{p}=\frac{e B}{4 \pi^{2}}\left[\sum_{\nu=0}^{\nu_{\max }^{(S=1)}} k_{f, \nu, 1}^{(p)}+\sum_{\nu=1}^{\nu_{(\max )}^{(S=-1)}} k_{f, \nu,-1}^{(p)}\right]
$$

and

$$
\begin{aligned}
\rho_{p}^{s} & =\frac{e B m_{p}^{*}}{2 \pi^{2}}\left[\sum_{\nu=0}^{\nu_{\max }^{(S=1)}} \frac{\sqrt{m_{p}^{* 2}+2 e B \nu}+\Delta_{p}}{\sqrt{m_{p}^{* 2}+2 e B \nu}} \ln \left|\frac{k_{f, \nu, 1}^{(p)}+E_{f}^{(p)}}{\sqrt{m_{p}^{* 2}+2 e B \nu}+\Delta_{p}}\right|\right. \\
& \left.+\sum_{\nu=1}^{\nu_{\max }^{(S=-1)}} \frac{\sqrt{m_{p}^{* 2}+2 e B \nu}-\Delta_{p}}{\sqrt{m_{p}^{* 2}+2 e B \nu}} \ln \left|\frac{k_{f, \nu,-1}^{(p)}+E_{f}^{(p)}}{\sqrt{m_{p}^{* 2}+2 e B \nu}-\Delta_{p}}\right|\right]
\end{aligned}
$$

where, $k_{f, \nu, \pm 1}^{(p)}$ are the Fermi momenta of protons for the Landau level, $\nu$ for the spin index, $S= \pm 1$, i.e. for spin up and spin down projections for the proton. These Fermi momenta are related to the Fermi energy of the proton as

$$
k_{f, \nu, S}^{(p)}=\sqrt{E_{f}^{(p)^{2}}-\left(\sqrt{m_{p}^{* 2}+2 e B \nu}+S \Delta_{p}\right)^{2}} .
$$

The number density and the scalar density of neutrons are given as

$$
\begin{aligned}
\rho_{n} & =\frac{1}{4 \pi^{2}} \sum_{S= \pm 1}\left\{\frac{2}{3} k_{f, S}^{(n)^{3}}+S \Delta_{n}\left[\left(m_{n}^{*}+S \Delta_{n}\right) k_{f, S}^{(n)}\right.\right. \\
& \left.\left.+E_{f}^{(n)^{2}}\left(\arcsin \left(\frac{m_{n}^{*}+S \Delta_{n}}{E_{f}^{(n)}}\right)-\frac{\pi}{2}\right)\right]\right\}
\end{aligned}
$$

and

$$
\rho_{n}^{s}=\frac{m_{n}^{*}}{4 \pi^{2}} \sum_{S= \pm 1}\left[k_{f, S}^{(n)} E_{f}^{(n)}-\left(m_{n}^{*}+S \Delta_{n}\right)^{2} \ln \left|\frac{k_{f, S}^{(n)}+E_{f}^{(n)}}{m_{n}^{*}+S \Delta_{n}}\right|\right] .
$$

The Fermi momentum, $k_{f, S}^{(n)}$ for the neutron with spin projection, $\mathrm{S}(S= \pm 1$ for the up (down) spin projection), is related to the Fermi energy for the neutron, $E_{f}^{(n)}$ as

$$
k_{f, S}^{(n)}=\sqrt{E_{f}^{(n)^{2}}-\left(m_{n}^{*}+S \Delta_{n}\right)^{2}} .
$$


In the equations (4)-(9, the parameter $\Delta_{i}$ is related to the to the anomalous magnetic moment for the nucleon, $i(i=p, n)$ as $\Delta_{i}=-\frac{1}{2} \kappa_{i} \mu_{N} B$, where, $\kappa_{i}$, occurring in the second term in the Lagrangian density given by Eq. (3) , is the value of the gyromagnetic ratio of the nucleon corresponding to the anomalous magnetic moment of the nucleon. Using the scalar densities of the nucleons in the presence of magnetic field, the values of the scalar fields, $\sigma, \zeta$ and $\delta$ are obtained by solving their coupled equations of motion,

The interaction Lagrangian modifying the masses of the $K$ and $\bar{K}$ mesons can be written as [22]

$$
\begin{aligned}
\mathcal{L}_{K}^{i n t} & =-\frac{i}{4 f_{k}^{2}}\left[\left(2 \bar{p} \gamma^{\mu} p+\bar{n} \gamma^{\mu} n\right)\left(K^{-}\left(\partial_{\mu} K^{+}\right)-\left(\partial_{\mu} K^{-}\right) K^{+}\right)\right. \\
& \left.+\left(\bar{p} \gamma^{\mu} p+2 \bar{n} \gamma^{\mu} n\right)\left(\overline{K^{0}}\left(\partial_{\mu} K^{0}\right)-\left(\partial_{\mu} \bar{K}^{0}\right) K^{0}\right)\right] \\
& +\frac{m_{K}^{2}}{2 f_{k}^{2}}\left[(\sigma+\sqrt{2} \zeta+\delta)\left(K^{+} K^{-}\right)+(\sigma+\sqrt{2} \zeta-\delta)\left(K^{0} \bar{K}^{0}\right)\right] \\
& -\frac{1}{f_{k}}\left[(\sigma+\sqrt{2} \zeta+\delta)\left(\partial_{\mu} K^{+}\right)\left(\partial^{\mu} K^{-}\right)+(\sigma+\sqrt{2} \zeta-\delta)\left(\partial^{\mu} K^{0}\right)\left(\partial^{\mu} \bar{K}^{0}\right)\right] \\
& +\frac{d_{1}}{2 f_{k}^{2}}\left[(\bar{p} p+\bar{n} n)\left(\left(\partial_{\mu} K^{+}\right)\left(\partial^{\mu} K^{-}\right)+\left(\partial_{\mu} K^{0}\right)\left(\partial^{\mu} \bar{K}^{0}\right)\right)\right] \\
& +\frac{d_{2}}{2 f_{k}^{2}}\left[\bar{p} p\left(\partial_{\mu} K^{+}\right)\left(\partial^{\mu} K^{-}\right)+\bar{n} n\left(\partial_{\mu} K^{0}\right)\left(\partial^{\mu} \bar{K}^{0}\right)\right]
\end{aligned}
$$

In the above, the first term is the vectorial Weinberg Tomozawa interaction term, which is the leading contribution in the chiral perturbation theory. This term is attractive for $\bar{K}$ mesons but repulsive for $K$ mesons. The next to leading contributions are given by the scalar meson exchange term (the second term) and the range term (the last three terms). The parameters $d_{1}$ and $d_{2}$ in the last two range terms are determined to be $2.56 / m_{K}$ and $0.73 / m_{K}$ respectively [22], by fitting to the empirical values of the kaon-nucleon $(\mathrm{KN})$ scattering lengths for $\mathrm{I}=0$ and $\mathrm{I}=1$ channels [63].

The Fourier transformations of the equations of motion for kaons (antikaons) obtained from the total Lagrangian density

$$
\mathcal{L}_{K}=\left(\partial_{\mu} \bar{K}\right)\left(\partial^{\mu} K\right)-m_{K(\bar{K})}^{2} \bar{K} K+\mathcal{L}_{K}^{i n t}
$$

lead to the dispersion relations,

$$
-\omega^{2}+|\vec{k}|^{2}+m_{K(\bar{K})}^{2}-\Pi_{K(\bar{K})}(\omega,|\vec{k}|)=0
$$


where $\Pi_{K(\bar{k})}$ denotes the kaon (antikaon) self energy in the medium. Explicitly, these self energies are given as

$$
\begin{gathered}
\Pi_{K}(\omega,|\vec{k}|)=-\frac{1}{4 f_{k}^{2}}\left[3\left(\rho_{p}+\rho_{n}\right) \pm\left(\rho_{p}-\rho_{n}\right)\right] \omega+\frac{m_{k}^{2}}{2 f_{k}}\left(\sigma^{\prime}+\sqrt{2} \zeta^{\prime} \pm \delta^{\prime}\right) \\
+\left[-\frac{1}{f_{k}}\left(\sigma^{\prime}+\sqrt{2} \zeta^{\prime} \pm \delta^{\prime}\right)+\frac{d_{1}}{2 f_{k}^{2}}\left(\rho_{p}^{s}+\rho_{n}^{s}\right)+\frac{d_{2}}{4 f_{k}^{2}}\left[\left(\rho_{p}^{s}+\rho_{n}^{s}\right) \pm\left(\rho_{p}^{s}-\rho_{n}^{s}\right)\right]\right]\left(\omega^{2}-|\vec{k}|^{2}\right)
\end{gathered}
$$

where the \pm signs refer to the $K^{+}$and $K^{0}$ respectively, and,

$$
\begin{gathered}
\Pi_{\bar{K}}(\omega,|\vec{k}|)=\frac{1}{4 f_{k}^{2}}\left[3\left(\rho_{p}+\rho_{n}\right) \pm\left(\rho_{p}-\rho_{n}\right)\right] \omega+\frac{m_{k}^{2}}{2 f_{k}}\left[\sigma^{\prime}+\sqrt{2} \zeta^{\prime} \pm \delta^{\prime}\right] \\
+\left[-\frac{1}{f_{k}}\left(\sigma^{\prime}+\sqrt{2} \zeta^{\prime} \pm \delta^{\prime}\right)+\frac{d_{1}}{2 f_{k}^{2}}\left(\rho_{p}^{s}+\rho_{n}^{s}\right)+\frac{d_{2}}{4 f_{k}^{2}}\left[\left(\rho_{p}^{s}+\rho_{n}^{s}\right) \pm\left(\rho_{p}^{s}-\rho_{n}^{s}\right)\right]\right]\left(\omega^{2}-|\vec{k}|^{2}\right)
\end{gathered}
$$

where the \pm signs refer to the $K^{-}$and $\bar{K}^{0}$ respectively. In the above, $\sigma^{\prime}, \zeta^{\prime}$ and $\delta^{\prime}$ are the deviations of the expectation values of the scalar fields from their vacuum expectation values. The masses for $\left.K(\bar{K}), m_{K(\bar{K})}^{*}=\omega(|\vec{k}|=0)\right)$, as modified due to their interactions with the nucleons and scalar mesons in the magnetized nuclear matter, are calculated from the dispersion relations given by equation (11), using the self energies for the kaons and antikaons given by equations (12) and (13). These mass modifications of the kaons and antikaons do not take into account the direct interaction of the charged kaons and antikaons with the electromagnetic field.

We consider the interaction of the medium modified charged $K^{ \pm}$mesons (due to interactions with the nucleons and scalar mesons in the magnetized nuclear matter) with mass $m_{K^{ \pm}}^{*}$, with the uniform magnetic field, $B$ through a minimal interacion, which leads to a further shift in the mass of the charged kaon (antikaon). In the presence of the uniform magnetic field, $B$, the mass squared of the spin zero charged kaon (antikaon), has summation of positive energy shifts of $(2 n+1) e B$ from the Landau levels (labelled by $n \geq 0$ ) [64 66]. Retaining only the lowest Landau level, i.e, $n=0$ level [43, 44], the effective mass of the charged $K^{ \pm}$meson in the magnetized nuclear medium, is given as

$$
m_{K^{ \pm}}^{e f f}=\sqrt{\left(m_{K^{ \pm}}^{*}\right)^{2}+e B}
$$




\begin{tabular}{|c|c|c|c|c|c|}
\hline \multirow{2}{*}{$e$} & \multicolumn{2}{|c|}{$\eta=0$} & \multicolumn{2}{c|}{$\eta=0.5$} \\
\cline { 2 - 6 }$e / m_{\pi}^{2}$ & $\rho_{B}=\rho_{0}$ & $\rho_{B}=4 \rho_{0}$ & $\rho_{B}=\rho_{0}$ & $\rho_{B}=4 \rho_{0}$ \\
\hline 0 & 521 & 544.6 & 518 & 528.9 \\
\hline 2 & (a) & 556.9 & 576.2 & 550.0 & 560.2 \\
\hline & (b) & 557.6 & 578.2 & 550.3 & 560.7 \\
\hline 4 & (a) & 589.7 & 604.9 & 584.2 & 593.6 \\
\hline & (b) & 590.2 & 612.8 & 584.4 & 596.6 \\
\hline 6 & (a) & 622.1 & 629.1 & 616.2 & 625.3 \\
\hline (b) & 622.1 & 645.5 & 616.9 & 632.0 \\
\hline 8 & (a) & 652.5 & 653.8 & 646.7 & 655.4 \\
\hline & (b) & 652.7 & 675.9 & 647.5 & 666.8 \\
\hline
\end{tabular}

TABLE I: In-medium masses for $K^{+}$meson in magnetized nuclear matter for densities of $\rho_{0}$ and $4 \rho_{0}$, asymmetric parameter, $\eta=0$ and 0.5 and for magnetic fields, $e B / m_{\pi}^{2}$ as $2,4,6$ and 8 . These masses are compared with the in-medium masses of $K^{+}$meson for zero magnetic field.

where, $m_{K^{ \pm}}^{*}$ is the mass of the $K^{ \pm}$meson arising due to interaction with the medium modified nucleons and scalar mesons, as has already been mentioned, is the solution for $\omega$ at $|\vec{k}|=0$ of the dispersion relation given by Eq. (11) for $K^{ \pm}$meson.

For the neutral kaons (antikaons), $K^{0}\left(\bar{K}^{0}\right)$, the effective masses are given as

$$
m_{K^{0}\left(\bar{K}^{0}\right)}^{e f f}=m_{K^{0}\left(\bar{K}^{0}\right)}^{*}
$$

In equations (14) and (15), $m_{K(\bar{K})}^{*}$ are the solutions for $\omega$ at $|\vec{k}|=0$ of the dispersion relations given by Eq. (11).

\section{RESULTS AND DISCUSSIONS}

The in-medium masses of the $K$ and $\bar{K}$ mesons are investigated in asymmetric nuclear matter in the presence of an external magnetic field, using a chiral SU(3) model. The medium modifications arise due to the interactions of these mesons with the protons, neutrons, and the scalar mesons $(\sigma, \zeta$, and $\delta)$. The charged $K$ and $\bar{K}$ mesons, i.e, $K^{ \pm}$mesons have additional 

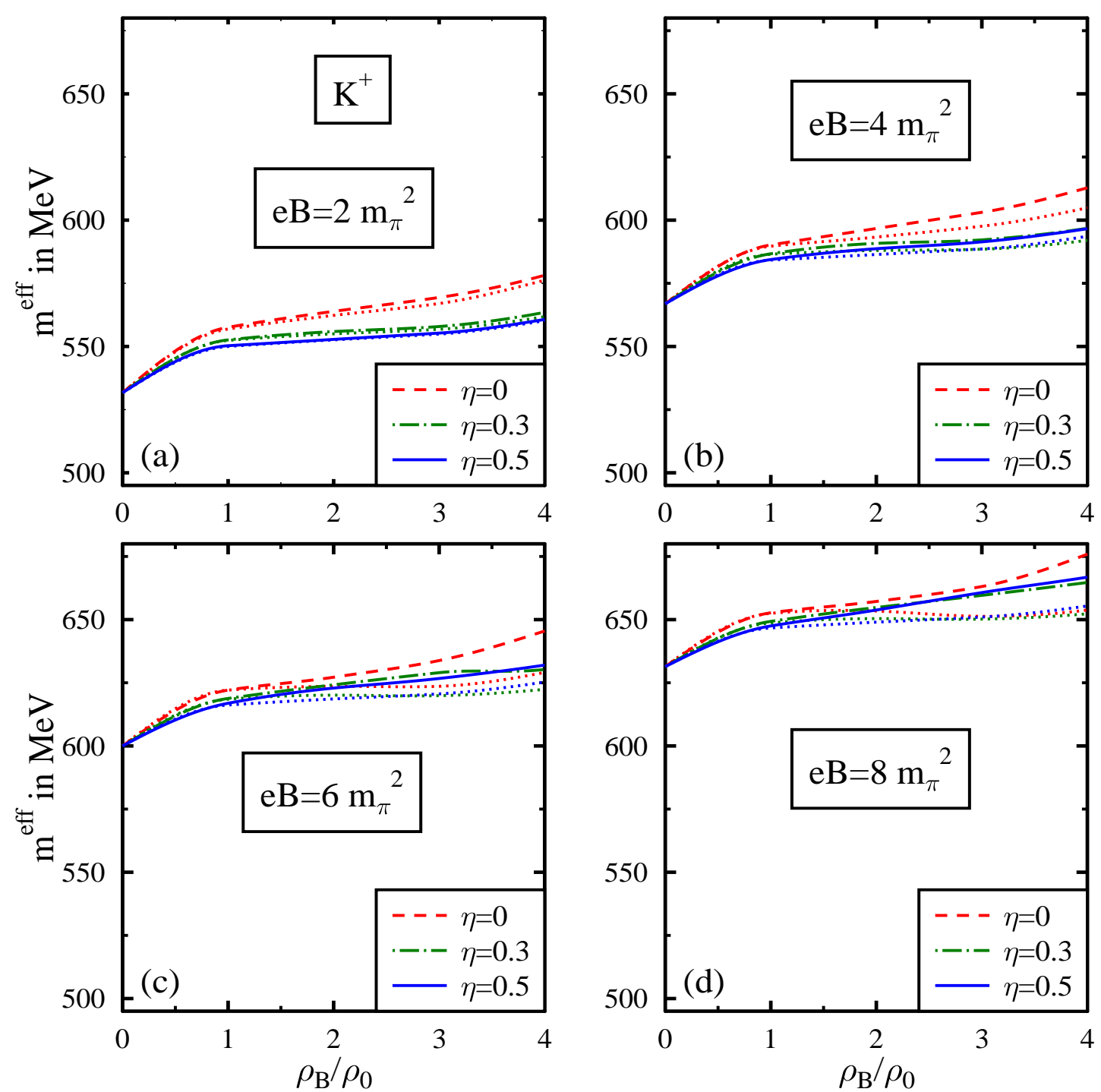

FIG. 1: (Color online) Effective mass of $K^{+}$(in $\mathrm{MeV}$ ) plotted as a function of the baryon density (in units of nuclear matter saturation density, $\rho_{0}$ ), with different values of isospin asymmetric parameter $\eta$, accounting for the effects of the anomalous magnetic moments (AMMs) of the nucleons. The results are compared with the case of not accounting for anomalous magnetic moments (shown as dotted lines).

mass modifications due to the Landau quantization effects in the presence of the external magnetic field, as given by Eq.(14). In the presence of an external magnetic field $\vec{B}=(0,0, B)$, the values of the scalar fields, $\sigma, \zeta$ and $\delta$ in the magnetized nuclear matter are solved from 

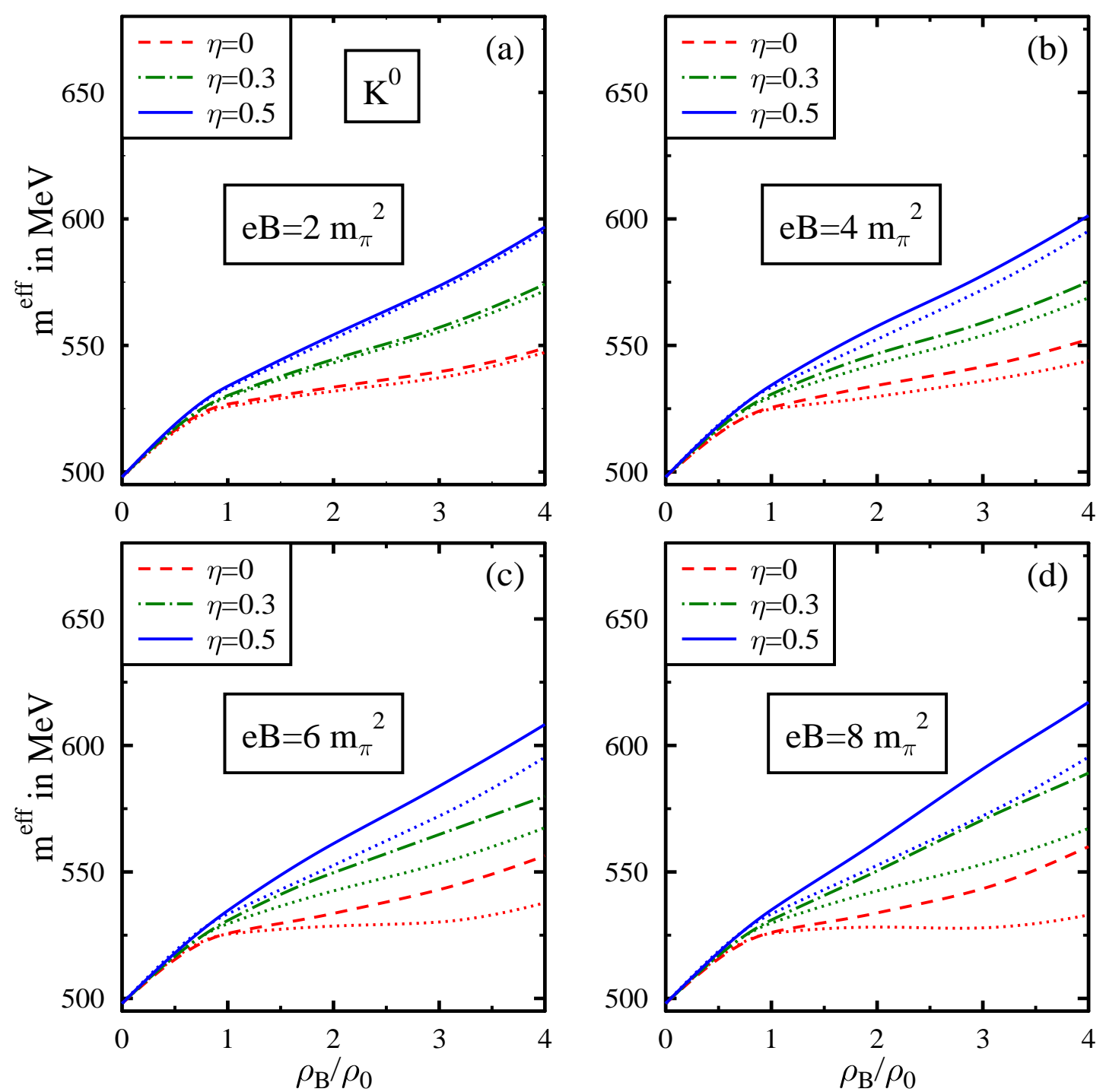

FIG. 2: (Color online) Effective mass of $K^{0}$ (in $\mathrm{MeV}$ ) plotted as a function of the baryon density (in units of nuclear matter saturation density, $\rho_{0}$ ), with different values of isospin asymmetric parameter $\eta$, accounting for the effects of the anomalous magnetic moments (AMMs) of the nucleons. The results are compared with the case of not accounting for anomalous magnetic moments (shown as dotted lines).

their equations of motion, for given values of the baryon density, $\rho_{B}$, the isospin asymmetry parameter, $\eta=\left(\rho_{n}-\rho_{p}\right) /\left(2 \rho_{B}\right)$ and the magnetic field, $B$. These coupled equations of motion are obtained in the mean field approximation (replacing the meson fields by their mean values), 

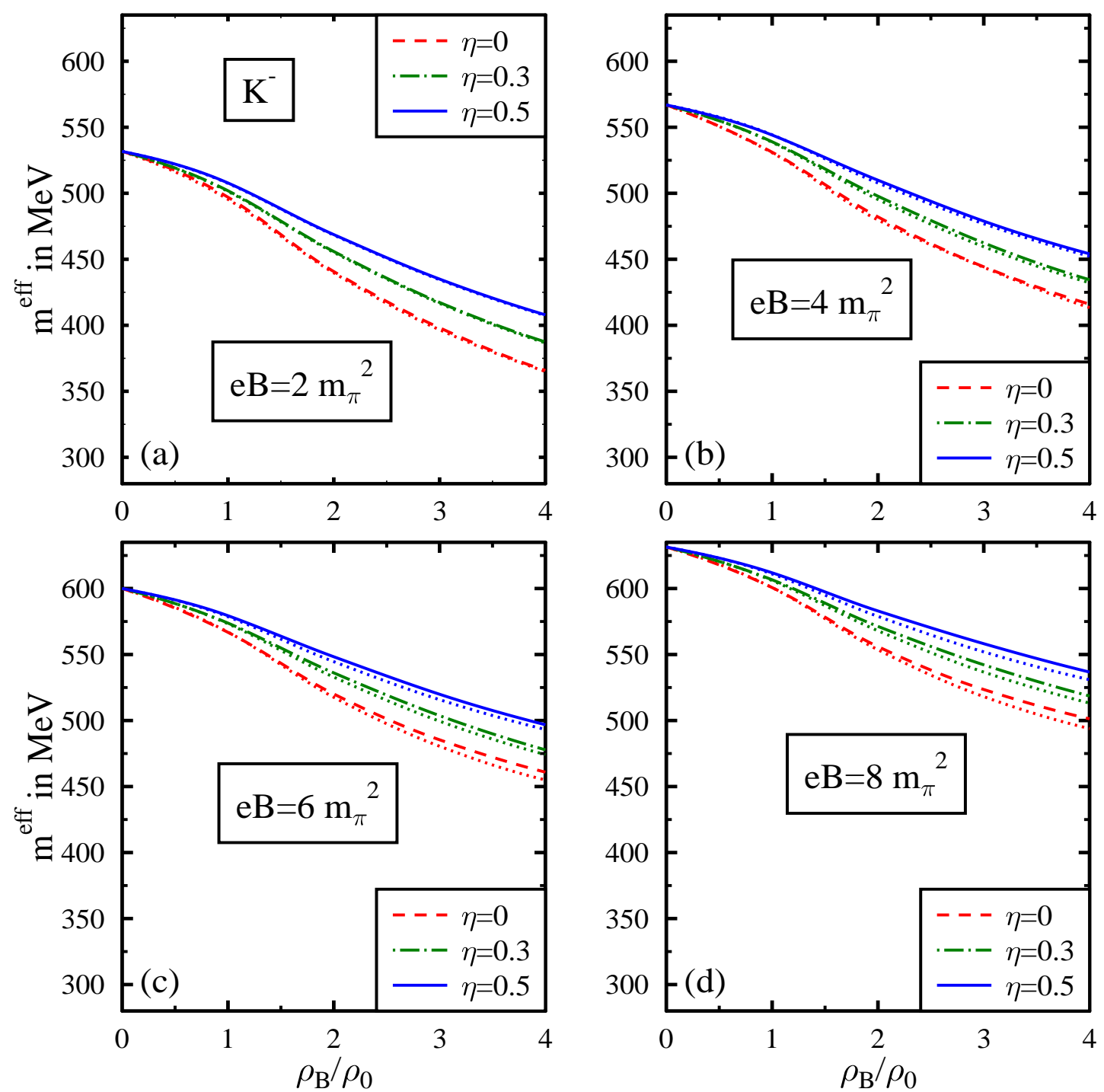

FIG. 3: (Color online) Effective mass of $K^{-}$(in $\mathrm{MeV}$ ) plotted as a function of the baryon density (in units of nuclear matter saturation density, $\rho_{0}$ ), with different values of isospin asymmetric parameter $\eta$, accounting for the effects of the anomalous magnetic moments (AMMs) of the nucleons. The results are compared with the case of not accounting for anomalous magnetic moments (shown as dotted lines).

along with the approximations $\bar{\psi}_{i} \psi_{j}=\delta_{i j}\left\langle\bar{\psi}_{i} \psi_{i}\right\rangle \equiv \delta_{i j} \rho_{i}^{s}$ and $\bar{\psi}_{i} \gamma^{\mu} \psi_{j}=\delta_{i j} \delta^{\mu 0}\left\langle\bar{\psi}_{i} \gamma^{0} \psi_{i}\right\rangle \equiv \delta_{i j} \delta^{\mu 0} \rho_{i}$, where, $\rho_{i}^{s}$ and $\rho_{i}$ are the scalar and number density of the nucleons $(i=\mathrm{p}, \mathrm{n})$. The number density and scalar density of the charged baryon, the proton, have contributions from the 

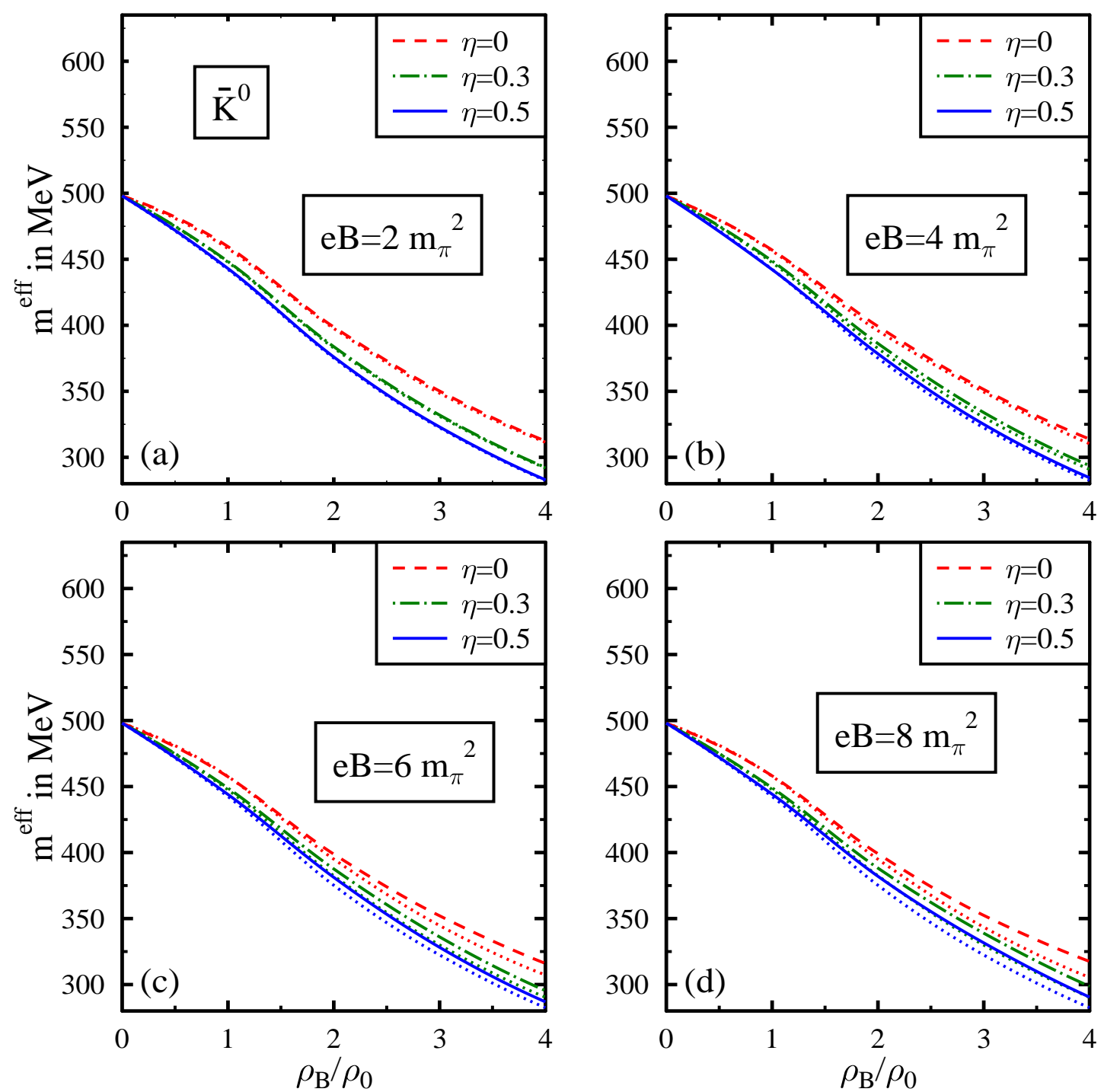

FIG. 4: (Color online) Effective mass of $\bar{K}^{0}$ (in $\mathrm{MeV}$ ) plotted as a function of the baryon density (in units of nuclear matter saturation density, $\rho_{0}$ ), with different values of isospin asymmetric parameter $\eta$, accounting for the effects of the anomalous magnetic moments (AMMs) of the nucleons. The results are compared with the case of not accounting for anomalous magnetic moments (shown as dotted lines).

Landau energy levels in the presence of the external magnetic field. The in-medium masses of the $K$ and $\bar{K}$ mesons are calculated by using the dispersion relations given by (11), with the self-energies for the kaons $\left(K^{+}, K^{0}\right)$ and antikaons $\left(K^{-}, \bar{K}^{0}\right)$ given by Eqs. (12) and (13) 


\begin{tabular}{|c|c|c|c|c|c|}
\hline \multirow{2}{*}{$e$} & \multicolumn{2}{|c|}{$\eta=0$} & \multicolumn{2}{c|}{$\eta=0.5$} \\
\cline { 2 - 6 }$e / m_{\pi}^{2}$ & $\rho_{B}=\rho_{0}$ & $\rho_{B}=4 \rho_{0}$ & $\rho_{B}=\rho_{0}$ & $\rho_{B}=4 \rho_{0}$ \\
\hline 0 & 524.8 & 547.1 & 529 & 591.5 \\
\hline 2 & (a) & 525.8 & 547.2 & 533.2 & 595.3 \\
\hline & (b) & 526.8 & 549.0 & 533.9 & 596.8 \\
\hline 4 & (a) & 524.8 & 543.9 & 533.4 & 595.2 \\
\hline & (b) & 525.5 & 552.4 & 534.3 & 601.3 \\
\hline 6 & (a) & 525.4 & 537.8 & 533.4 & 595.3 \\
\hline & (b) & 525.7 & 556.4 & 534.7 & 608.3 \\
\hline 8 & (a) & 525.7 & 533.0 & 533.4 & 595.4 \\
\hline & (b) & 526.1 & 560.0 & 535.0 & 617.1 \\
\hline
\end{tabular}

TABLE II: In-medium masses for $K^{0}$ meson in magnetized nuclear matter for densities of $\rho_{0}$ and $4 \rho_{0}$, asymmetric parameter, $\eta=0$ and 0.5 and for magnetic fields, $e B / m_{\pi}^{2}$ as $2,4,6$ and 8 . These masses are compared with the in-medium masses of $K^{0}$ meson for zero magnetic field.

respectively. As has already been mentioned the charged $K^{ \pm}$mesons have additional mass shifts due to direct interaction with the electromagnetic field.

The effective massses of the kaons $\left(K^{+}, K^{0}\right)$ and the antikaons $\left(K^{-}\right.$and $\left.\bar{K}^{0}\right)$ in the magnetized asymmetric nuclear matter, are plotted as functions of $\rho_{B} / \rho_{0}$ (the baryon density in units of the nuclear matter saturation density), in Figs. 1, 2, 3 and 4. These are shown for various values of the isospin asymmetry parameter $\eta$, including the effects from the anomalous magnetic moments (AMMs) of the nucleons. These are compared with the case that does not account for the effects from the anomalous magnetic moments (shown as dotted lines). At $\rho_{B}=0$, there are still mass modifications of charged kaons (antikaons), i.e, $K^{ \pm}$mesons, due to direct interaction with the electromagnetic field, as given by Eq. (14), while for the electrically charged neutral $K^{0}$ and $\left(\bar{K}^{0}\right)$ mesons, there is no such mass modification due to magnetic field at $\rho_{B}=0$. For the isospin asymmetric nuclear matter with the isospin asymmetry parameter, $\eta=0.5$, the medium comprises of only neutrons, and hence the only effect of magnetic field is due to the anomalous magnetic moment (AMM) of the neutrons. Hence, in the case when the 


\begin{tabular}{|c|c|c|c|c|c|}
\hline \multirow{2}{*}{$e$} & \multicolumn{2}{|c|}{$\eta=0$} & \multicolumn{2}{c|}{$\eta=0.5$} \\
\cline { 2 - 6 }$e / m_{\pi}^{2}$ & $\rho_{B}=\rho_{0}$ & $\rho_{B}=4 \rho_{0}$ & $\rho_{B}=\rho_{0}$ & $\rho_{B}=4 \rho_{0}$ \\
\hline \multicolumn{2}{|c|}{0} & 452 & 307.9 & 471.9 & 360.2 \\
\hline 2 & (a) & 495.4 & 365 & 507.5 & 407.4 \\
\hline & (b) & 497.0 & 365.8 & 507.8 & 407.9 \\
\hline 4 & (a) & 530.7 & 413.4 & 544.3 & 452.3 \\
\hline & (b) & 531.1 & 416.0 & 544.1 & 454.1 \\
\hline 6 & (a) & 567.0 & 455.0 & 578.6 & 439.2 \\
\hline & (b) & 566.8 & 460.9 & 579.4 & 496.8 \\
\hline 8 & (a) & 600.7 & 493.9 & 611.0 & 530.9 \\
\hline & (b) & 600.7 & 501.3 & 611.9 & 536.7 \\
\hline
\end{tabular}

TABLE III: In-medium masses for $K^{-}$meson in magnetized nuclear matter for densities of $\rho_{0}$ and $4 \rho_{0}$, asymmetric parameter, $\eta=0$ and 0.5 and for magnetic fields, $e B / m_{\pi}^{2}$ as $2,4,6$ and 8 . These masses are compared with the in-medium masses of $K^{-}$meson for zero magnetic field.

AMM effects are not taken into consideration, the expectaion values of the scalar fields remain independent of the magnetic field.

For isospin symmetric nuclear matter $\left(\rho_{p}=\rho_{n}\right)$, in the absence of a magnetic field, the scalar densities of proton and neutron are identical and the value of the scalar isovector meson, $\delta$ remains zero. The masses of the kaons and antikaons are calculated from the dispersion relation given by equation (11), with additional positive shifts for the charged $K^{ \pm}$, arising from the direct interaction of these mesons with the magnetic field through minimal coupling. The first term of the self energy, the Weinberg Tomozawa term, leads to a rise (drop) in the mass of the kaon (antikaon). The second term, which is the scalar exchange term is attractive for both the kaons and antikaons. The last three terms in the self energy comprise the range terms, of which, the first term is repulsive, whereas the second and third range terms (the $d_{1}$ and $d_{2}$ terms) are attractive for both kaons and antikaons. It might be noted here that in the presence of a magnetic field, due to the different interactions of the proton (charged nucleon) and the neutron with the magnetic field, the value of $\delta\left(\sim\left(\rho_{s}^{p}-\rho_{n}^{s}\right)\right)$ no longer remains zero. 


\begin{tabular}{|c|c|c|c|c|c|}
\hline \multirow{2}{*}{$e$} & \multicolumn{2}{|c|}{$\eta=0$} & \multicolumn{2}{c|}{$\eta=0.5$} \\
\cline { 2 - 6 }$e / m_{\pi}^{2}$ & $\rho_{B}=\rho_{0}$ & $\rho_{B}=4 \rho_{0}$ & $\rho_{B}=\rho_{0}$ & $\rho_{B}=4 \rho_{0}$ \\
\hline 0 & 455.9 & 310.9 & 437.9 & 278.6 \\
\hline 2 & (a) & 458.1 & 311.4 & 442.3 & 282.5 \\
\hline & (b) & 459.6 & 312.4 & 443.0 & 283.0 \\
\hline 4 & (a) & 456.3 & 310.3 & 442.4 & 282.5 \\
\hline & (b) & 457.0 & 313.8 & 442.2 & 284.5 \\
\hline 6 & (a) & 457.6 & 307.3 & 442.4 & 282.5 \\
\hline & (b) & 457.4 & 316.0 & 444.0 & 286.9 \\
\hline 8 & (a) & 457.8 & 305.4 & 442.4 & 282.5 \\
\hline & (b) & 458.1 & 317.5 & 444.2 & 290.5 \\
\hline
\end{tabular}

TABLE IV: In-medium masses for $\bar{K}^{0}$ meson in magnetized nuclear matter for densities of $\rho_{0}$ and $4 \rho_{0}$, asymmetric parameter, $\eta=0$ and 0.5 and for magnetic fields, $e B / m_{\pi}^{2}$ as $2,4,6$ and 8 . These masses are compared with the in-medium masses of $\bar{K}^{0}$ meson for zero magnetic field.

The coupled equations of motion for $\sigma, \zeta$ and $\delta$ are solved to obtain the values of these fields (and hence the nucleon scalar densities), which are used to calculate the masses of the kaons and antikaons in the magnetized nuclear matter. The contributions due to isospin asymmetry in the medium, arise explicitly from the last terms of the Weinberg Tomozawa term, the scalar exchange term, and the first and last range terms. There is contribution from isospin asymmetry also from the second range term implicitly, as the values of the scalar densities of the proton and neutron are different in the isospin asymmetric medium (as calculated from the coupled equations of motion for $\sigma, \zeta$ as well as $\delta$ ), as compared to in symmetric nuclear matter $(\eta=0)$.

In isospin symmetric nuclear matter $(\eta=0)$, as can be seen from figures 1 and 2 , the masses of both $K^{+}$as well as $K^{0}$ are observed to increase as baryon density is increased, with an initial rapid rise upto a density of about nuclear matter saturation density, $\rho_{0}$. Such a behaviour for the kaons was also observed for the zero magnetic field [22]. For isospin symmetric nuclear matter, the increase in the masses of the kaons is due to the increase due to the Weinberg 
Tomozawa term and the first range term, which dominates over the drop due to the scalar exchange and the last two range terms. In the presence of magnetic field, there is an additional positive shift in the mass of $K^{+}$(given by equation (14)), as can be seen in figure 1 as well as from Table I, where the in-medium masses of $K^{+}$have been given for different magnetic fields ((a) without and (b) with the AMM effects) both for isospin symmetric nuclear matter, as well as, for asymmetric nuclear matter with $\eta=0.5$. The effects of anomalous magnetic moments (AMM) of the nucleons are observed to have larger masses for both $K^{+}$and $K^{0}$, as compared to when these are not taken into account, as can be observed form the figures 1 and 2, as well as, seen from Tables I and II. The values of these kaon masses at different magnetic fields can be compared with the values for zero magnetic field. As can be seen for the neutral kaon $K^{0}$ from Table II, the mass is observed to increase by maximum value of about 1 (6) $\mathrm{MeV}$ at $\rho_{B}=\rho_{0}$ from the zero magnetic field case, for the symmetric (asymmetric) nuclear matter, for the magnetic fields considered in the present investigation, and by a maximum increase of about $13(25) \mathrm{MeV}$ at $\rho_{B}=4 \rho_{0}$. The neutral $K^{0}$ meson has been studied recently in the presence of magnetic field for electrically charge neutral matter, using the chiral $\mathrm{SU}(3)$ model as used in the present investigation and including one loop corrections to the kaon propagator [67]. There is observed to be a similar rise in the mass of $K^{0}$ as has been obtained in the present work.

The isospin asymmetry contribution to the mass of the $K^{+}\left(K^{0}\right)$ is negative (positive) from the Weinberg Tomozawa term. This leads to a smaller (larger) modification to the mass of the $K^{+}\left(K^{0}\right)$ mass, due to the vectorial interaction with the nucleons. The contributions from the $\delta$ term in the scalar exchange and the first range term are positive (negative) for $K^{+}$meson and of the opposite sign for $K^{0}$ meson. The contribution from $\delta$ term on the mass of the $K^{+}\left(K^{0}\right)$ in the isospin asymmetric medium turns out to be negative (positive) as the contribution from the first range term dominates over the scalar exchange term. The drop due to the last term ( $d_{2}$ term) is lessened (enhanced) due to the isospin asymmetric part, as can be seen from the expression for the self energy for kaon doublet, given by equation (12). The isospin asymmetry is observed to diminish (enhance) the increase in the mass of the $K^{+}\left(K^{0}\right)$ predominantly due to the contributions from the Weinberg Tomozawa and the first range term. These are observed in figures 1 and 2, as strong dependence of the $K^{0}$ mass on isospin asymmetry, whereas the 
mass of $K^{+}$is observed to be rather insensitive to the isospin asymmetry. These can also be seen from the values of the masses of the kaon doublet given in Tables I and II, where the contribution from the lowest Landau level (due to direct interaction with the electromagnetic field), in mass of the charged $K^{+}$, has been taken into account. The antikaons are observed to have large drop in isospin symmetric nuclear matter, in their masses, as can be observed from figures 3 anf 4, as well as from their values as listed in Tables III and IV. This is due to the attractive interactions of the Weinberg-Tomozawa term, the scalar exchange term as well as from the last two range terms, which dominate over the repulsive interaction of the first range term. The isospin asymmetry effects lead to larger (smaller) masses for the $K^{-}\left(\bar{K}^{0}\right)$ due to opposing effects arising from the vectorial, scalar exchage and the first and last range terms of the self energy for the antikaons. The isospin effects are observed to show monotonous behaviour for the masses of the antikaons, as well as for $K^{0}$. However, the isospin effects on the $K^{+}$mass is observed to be rather small and are observed not to follow a monotonic behaviour as the other mesons (antikaons and $K^{0}$ meson), in the present investigation.

The values of the masses of the antikaons $\left(K^{-}\right.$and $\left.\bar{K}^{0}\right)$ have positive shifts in the presence of the magnetic field as compared to zero magnetic field. In isospin symmetric nuclear matter, for $e B=2 m_{\pi}^{2}\left(8 m_{\pi}^{2}\right)$, the value of $K^{-}$mass undergoes a modification from the value of 452 $\mathrm{MeV}$ at zero magnetic field to 497 (600.7) $\mathrm{MeV}$ at $\rho_{B}=\rho_{0}$ and 365.8 (501.3) $\mathrm{MeV}$ at $\rho_{B}=4 \rho_{0}$, when the AMM effects of nucleons are taken into consideration. The isospin asymmetry is observed to lead to larger values of the $K^{-}$mass as compared to the isospin symmetric case, and the mass shifts for $\eta=0.5$ (as compared to $\eta=0$ ), are upto a maximum value of around $15 \mathrm{MeV}$ at $\rho_{B}=\rho_{0}$ and around $42 \mathrm{MeV}$ at $\rho_{B}=4 \rho_{0}$ for the values of the magnetic fields considered in the present investigation. The effects of isospin asymmetry are thus observed to be quite negligible, compared to the medium modifications of $K^{-}$mass with baryon density. The values of the $K^{-}$are smaller when the AMM effects of the nucleons are not taken into account and the modifications arising from the AMM effects are observed to be marginal. For $\bar{K}^{0}$, the mass modification is an increase upto a maximum value of about 2 (6) MeV from the zero magnetic field case at $\rho_{B}=\rho_{0}$, and about $6(12) \mathrm{MeV}$ at $\rho_{B}=4 \rho_{0}$ for $\eta=0(0.5)$ for the values of magnetic field considered in the present investigation. The effect of the baryon density on the masses of the antikaons (plotted in figures 3 and 4), is thus observed to be the 
most dominant effect as compared to the effects from the isospin asymmetry and AMMs of the nucleons.

The effective masses of the kaons and antikaons, arise from the values of the scalar fields, $\sigma, \zeta$ and $\delta$, which are solved to obtain the values of the scalar densities of the nucleons. The charged mesons, in addition, have a positive mass shift, due to interaction with the electromagnetic field. The modifications of the scalar mesons are crucial for the medium modifications of the kaons and anikaons in the magnetized nuclear matter. The effects of anomalous magnetic moments (AMM) of the nucleons are incorporated in the expressions for the scalar and number densities of the nucleons, while solving the scalar fields. These effects are observed to be more dominant for the $\sigma$ and $\zeta$ fields, as compared to for the isovector scalar $\delta$ field as has been studied in Ref. [43]. The magnitudes of the scalar fields are observed to be smaller (leading to larger values for the fluctuations in these fields from the vacuum and hence the contributions from these terms are larger in magnitude) when the AMM effects are not taken into account, as compared to when these effects are considered. Hence, the AMM effects are observed to lead to smaller masses for the kaons and antikaons, as compared to when these effects are not considered. The AMM effects are most prominently observed in the in-medium mass of $K^{0}$, plotted in figure 2, where, the magnitudes in the modifications of the masses are observed to be of similar order for the values of isospin asymmetry parameters $(\eta=0,0.3$ and 0.5$)$ as shown

in the figure. This is because the modifications of the values of $\sigma$ and $\zeta$ are much larger as compared to the value of $\delta$, due to AMM effects [43]. However, the mass modifications of $K^{0}$ meson, due to AMM effects are observed to be smaller when the isospin asymmetry of the medium is increased, as can be also seen from their values given in Table II as well as from figure 2. The AMM effects for the mass of $K^{0}$ meson, are more dominant for the higher values of the magnetic field ( $e B=6 m_{\pi}^{2}$ and $e B=8 m_{\pi}^{2}$, shown in (c) and (d) of figure 2), as expected.

\section{SUMMARY}

To summarize, we have calculated the in-medium masses of the kaons and antikaons in asymmetric nuclear matter in the presence of an external magnetic field, using a chiral SU(3) model. The modifications of the masses of these strange pseudoscalar mesons arise due to the leading and next to leading order terms within the chiral perturbative theory. The leading 
contribution (the Weinberg Tomozawa term) gives a drop (rise) of the antikaons (kaons) due to the attractive (repulsive) interaction in the nuclear matter. The next to leading contributions are due to the scalar exchange and the range terms. The effects of the anomalous magnetic moments (AMM) of the nucleons are taken into consideration in the present work. There is observed to be rise (drop) in the in-medium masses of the kaons and the antikaons with density for given isospin asymmetry and magetic fields. For the charged $K^{ \pm}$mesons, there are additionl positive shifts in the mass in the presence of magnetic field, from the lowest Landau level in the present investigation. The isospin effetcs are observed to be large at high densities, and the effect is observed to be much more prominent for $K^{0}$ mass as compared to the antikaons, and $K^{+}$masses. The AMM effcts are observed to be significant for the mass of $K^{0}$, expecially for higher values of the magnetic fields. The density effects are, however, observed to be the dominant medium effects as compared to the effects from the magnetic field and isospin asymmetry of the magnetized nuclear medium. The mass modification of $K^{0}$ meson observed to have large isospin asymmetry as well as AMM effects, especially at high densities, as compared to these effects for the $K^{+}$, as well as, $K^{-}$and $\bar{K}^{0}$ mesons, should have observable consequences on the $K^{+} / K^{0}$ ratio in the high energy asymmetric nuclear collisions.

One of the authors (AM) is grateful to ITP, University of Frankfurt, for warm hospitality and acknowledges financial support from Alexander von Humboldt foundation when this work was initiated.

[1] V. Skokov, A. Y. Illarionov and V. Toneev, Int. J. Mod. Phys. A 24, 5925 (2009).

[2] W. T. Deng and X.G.Huang, Phys.Rev. C 85, 044907 (2012).

[3] D. Kharzeev, L. McLerran and H. Warringa, Nucl. Phys. A 803, 227 (2008).

[4] K. Fukushima, D. E. Kharzeev and H. J. Warringa, Phys. Rev. D 78, 074033 (2008).

[5] M. A. Shifman, A. I. Vainshtein and V. I. Zakharov, Nucl. Phys. B 147, 385 (1979).

[6] M. A. Shifman, A. I. Vainshtein and V. I. Zakharov, Nucl. Phys. B 147, 448 (1979).

[7] B. D. Serot and J. D. Walecka, Adv. Nucl. Phys. 16, 1 (1986).

[8] S. A. Chin, Ann. Phys. (N.Y.) 108, 301 (1977).

[9] K. Tsushima, D.H. Lu, A.W. Thomas, K. Saito, R.H. Landau, Phys. Rev. C 59, 2824 (1999). 
[10] A. Sibirtsev, K. Tsushima, A.W. Thomas, Eur. Phys. J. A 6, 351 (1999).

[11] M. Lutz, Phys. Lett. B 426, 12 (1998).

[12] M. Lutz and E. E. Kolomeitsev, Nucl. Phys. A 700, 193 (2002).

[13] M. Lutz and C. L. Korpa, Nucl. Phys. A 700, 309 (2002).

[14] A. Ramos and E. Oset, Nucl. Phys. A 671, 481 (2000).

[15] L. Tolós, A. Ramos, and A. Polls, Phys. Rev. C 65, 054907 (2002).

[16] L. Tolós, A. Ramos, A. Polls, and T.T.S. Kuo, Nucl. Phys. A 690, 547 (2001).

[17] P. Papazoglou, D. Zschiesche, S. Schramm, J. Schaffner-Bielich, H. Stöcker, and W. Greiner, Phys. Rev. C 59, 411 (1999).

[18] A. Mishra, K. Balazs, D. Zschiesche, S. Schramm, H. Stöcker and W. Greiner, Phys. Rev. C 69, 024903 (2004).

[19] A. Mishra, E. L. Bratkovskaya, J. Schaffner-Bielich, S. Schramm and H. Stöcker, Phys. Rev. C 70, 044904 (2004).

[20] A. Mishra and S. Schramm, Phys. Rev. C 74, 064904 (2006).

[21] A. Mishra, S. Schramm and W. Greiner, Phys. Rev. C 78, 024901 (2008).

[22] Amruta Mishra, Arvind Kumar, Sambuddha Sanyal, S. Schramm, Eur. Phys, J. A 41, 205 (2009).

[23] D. Zschiesche, A. Mishra, S. Schramm, H. Stöcker and W. Greiner, Phys. Rev. C 70, 045202 (2004).

[24] Amruta Mishra, Phys. Rev. C 91, 035201 (2015).

[25] A. Mishra, A. Kumar, S. Sanyal, V. Dexheimer, S. Schramm, Eur. Phys. Jour. A 45, 169 (2010).

[26] A. Mishra, E. L. Bratkovskaya, J. Schaffner-Bielich, S.Schramm and H. Stöcker, Phys. Rev. C 69, 015202 (2004).

[27] Amruta Mishra and Arindam Mazumdar, Phys. Rev. C 79, 024908 (2009).

[28] Arvind Kumar and Amruta Mishra, Phys. Rev. C 81, 065204 (2010).

[29] Arvind Kumar and Amruta Mishra, Eur. Phys. J. A 47, 164 (2011).

[30] Divakar Pathak and Amruta Mishra, Adv. High Energy Phys. 2015, 697514 (2015).

[31] Divakar Pathak and Amruta Mishra, Phys. Rev. C 91, 045206 (2015).

[32] Divakar Pathak and Amruta Mishra, Int. J. Mod. Phy. E 23, 1450073 (2014).

[33] Arvind Kumar and Amruta Mishra, Phys. Rev. C 82, 045207 (2010). 
[34] Amruta Mishra and Divakar Pathak, Phys. Rev. C 90, 025201 (2014).

[35] A. Le Yaouanc, L. Oliver, O. Pene and J. C. Raynal, Phys. Rev. D 8, 2223 (1973).

[36] A. Le Yaouanc, L. Oliver, O. Pene and J. C. Raynal, Phys. Rev. D 9, 1415 (1974);

[37] A. Le Yaouanc, L. Oliver, O. Pene and J. C. Raynal, Phys. Rev. D 11, 1272 (1975);

[38] T. Barnes, F. E. Close, P. R. Page and E. S. Swanson, Phys. Rev. D 55, 4157 (1997). (2002).

[39] B. Friman, S. H. Lee and T. Song, Phys. Lett, B 548, 153 (2002).

[40] Amruta Mishra, S. P. Misra and W. Greiner, Int. J. Mod. Phys. E 24, 155053 (2015).

[41] Amruta Mishra and S. P. Misra, Phys. Rev. C 95, 065206 (2017).

[42] Ankit Kumar, Pallabi Parui, Sourodeep De, Amruta Mishra, arXiv: 1811.12570 (nucl-th).

[43] Sushruth Reddy P., Amal Jahan C.S., Nikhil Dhale, Amruta Mishra, J. Schaffner-Bielich, Phys. Rev. C 97, 065208 (2018).

[44] Nikhil Dhale, Sushruth Reddy P., Amal Jahan C.S., Amruta Mishra, Phys. Rev. C 98, 015202 (2018).

[45] Amal Jahan C.S., Nikhil Dhale, Sushruth Reddy P., Shivam Kesarwani and Amruta Mishra, Phys. Rev. C 98, 065202 (2018).

[46] Amal Jahan C.S., Shivam Kesarwani, Sushruth Reddy P., Nikhil Dhale, and Amruta Mishra, arXiv:1807.07572 (nucl-th).

[47] Pallabi Parui, Ankit Kumar, Sourodeep De, Amruta Mishra, arXiv: 1811.04622 (nucl-th).

[48] A. Broderick, M. Prakash and J.M.Lattimer, Astrophys. J. 537, 351 (2002).

[49] A.E. Broderick, M. Prakash and J. M. Lattimer, Phys. Lett. B 531, 167 (2002).

[50] F. X. Wei, G. J. Mao, C. M. Ko, L. S. Kisslinger, H. Stöcker, and W. Greiner, J. Phys. G, Nucl. Part. Phys. 32, 47 (2006).

[51] Guang-Jun Mao, Akira Iwamoto, Zhu-Xia Li, Chin. J. Astrophys. 3, 359 (2003).

[52] M. Pitschmann and A. N. Ivanov, arXive : 1205.5501 (math-ph).

[53] V. Dexheimer, R. Negreiros, S. Schramm, Eur. Phys. Journal A 48, 189 (2012).

[54] V. Dexheimer, B. Franzon and S. Schramm, Jour. Phys. Conf. Ser. 861, 012012 (2017).

[55] R. M. Aguirre and A. L. De Paoli, Eur. Phys. J. A 52, 343 (2016).

[56] S.Weinberg, Phys. Rev. 1661568 (1968).

[57] S. Coleman, J. Wess, B. Zumino, Phys. Rev. 1772239 (1969). 
[58] C.G. Callan, S. Coleman, J. Wess, B. Zumino, Phys. Rev. 1772247 (1969).

[59] W. A. Bardeen and B. W. Lee, Phys. Rev. 1772389 (1969).

[60] J. Schechter, Phys. Rev. D 21, 3393 (1980).

[61] J.Ellis, Nucl. Phys. B 22, 478 (1970).

[62] B. A. Campbell, J. Ellis and K. A. Olive, Nucl. Phys. B 345, 57 (1990).

[63] T. Barnes and E. S. Swanson, Phys. Rev. C 49, 1166 (1994).

[64] M. N. Chernodub, Phys. Rev. D 82, 085011 (2010).

[65] M. N. Chernodub, Lect. Notes Phys. 871, 143 (2013).

[66] R. M. Aguirre, Phys. Rev. D 95, 074029 (2017).

[67] R. M. Aguirre, Eur. Phys. J. A 55, 28 (2019). 